\title{
DIFFUSION AND HEAT FLOW EQUATIONS FOR THE MID-LATITUDE TOPSIDE IONOSPHERE
}

\author{
J.-P. ST.-MAURICE \\ Department of Atmospheric and Oceanic Science, The University of Michigan, Ann Arbor, MI 48109, U.S.A. \\ and \\ K. w. SCHUNK \\ Aeronomy Center, Utah State University, Logan, UT 84322, U.S.A.
}

(Received in final form 25 January 1977)

\begin{abstract}
For application to the mid-latitude topside ionosphere, we have derived diffusion and heat flow equations for a gas mixture composed of two major ions, electrons and a number of minor ions. These equations were derived by expanding the velocity distribution of each constituent about its 13 lower order velocity moments. As a consequence, each constituent was allowed to have its own temperature and drift velocity. The restriction to mid-latitudes results because we have assumed that the species temperature and drift velocity differences were small. In deriving the diffusion and thermal conduction equations, we have discovered some new transport effects. For the major ions, we have found that: (1) a temperature gradient in either gas causes thermal diffusion in both gases; (2) a temperature gradient in either gas causes heat to flow in both gases; and (3) a relative drift between the major ion gases induces a heat flow in both gases. Similar transport effects have also been found for the minor ions.
\end{abstract}

\section{INTRODUCTION}

Early studies of the topside ionosphere by Johnson (1960), Mange (1960), Kockarts and Nicolet (1963), Bauer (1966), and others, gave special emphasis to a static nonflow model where the principal topside ions $\left(\mathrm{O}^{+}, \mathrm{H}^{+}, \mathrm{He}^{+}\right)$arranged themselves in stratified layers according to ion mass. This static or diffusive equilibrium model of the mid-latitude topside ionosphere was later extended by Walker (1967) and Schunk and Walker (1969) to include thermal diffusion effects. In Schunk and Walker (1969), diffusive equilibrium density profiles were presented for a topside ionosphere with two major ions, electrons, and a number of minor ions, and it was shown that thermal diffusion exerts an important influence on the altitude distribution of these species.

With mounting experimental observations of a significant ionization flow between the ionosphere and the protonosphere (Vasseur and Waldteufel, 1968; Evans, 1969; Evans et al., 1970; Behnke, 1970; Evans, 1971a, b; Hagen, 1972), later theoretical studies of the mid-latitude topside ionosphere were concerned with more dynamic models which took account of the relative flow between the interacting species (Banks et al., 1971; Mayr et al., 1972; Nagy and Banks, 1972; Moffett and Murphy, 1973; Massa et al., 1974; Murphy et al., 1976). These studies indicated that the ioniza- tion flow between the ionosphere and protonosphere is strong enough to appreciably affect the $F$ region and topside ionosphere. However, in all of these studies except Murphy et al. (1976), the flow of ions and electrons along the geomagnetic field has been described by the binary diffusion formulation of Chapman and Cowling (1970). Although the binary formulation is convenient to use, thermal diffusion effects are neglected. Furthermore, $F$ region studies by Schunk and Walker $(1970 a, b)$ using a more rigorous formulation of multispecies transport indicate that the binary diffusion coefficients may be in error by up to a factor of two when Coulomb collisions dominate.

Schunk and Walker $(1970 \mathrm{a}, \mathrm{b})$ used the multicomponent formulation of Hirschfelder et al. (1964) to derive the ordinary and thermal diffusion coefficients. In this formulation, these diffusion coefficients are expressed as ratios of determinants, with the size of the determinants depending upon both the number of species in the gas mixture and the level of approximation. Therefore, as the number of species in the gas mixture increases, the effort involved in evaluating the diffusion coefficients increases rapidly if these coefficients are to be accurate to within $20 \%$ (cf. Schunk and Walker, $1970 a, b)$. Furthermore, this approach has an added disadvantage in that the different species are assumed to have a common temperature. Since ionospheric modeling has reached the point where 
the different temperatures of the various ions are taken into consideration (Roble and Hastings, 1977), it would be useful to have a transport theory that takes account of this feature.

An alternative approach to the derivation of transport equations for the Earth's upper atmosphere and ionosphere has recently been presented by Schunk (1975). This approach, which is based on the work of Grad $(1949,1958)$ and Burgers (1969), yields a continuity, momentum, internal energy, stress tensor and heat flow equation for each species in the gas mixture and, therefore, provides a better description of transport for situations where the components of the gas mixture have separate flow velocities and temperatures. Furthermore, this approach has the added advantage in that ordinary diffusion, thermal diffusion and thermoelectric transport are included at a level of approximation that is equivalent to that obtained by Schunk and Walker (1970a, b) from the multicomponent formulation of Hirschfelder et al. (1964).

In the present investigation, the general system of transport equations presented by Schunk (1975) is applied to the mid-latitude topside ionosphere. In this region of the ionosphere, the general system of transport equations reduces significantly, and it is possible to derive relatively simple diffusion and heat flow equations for a plasma composed of two major ions, electrons and a number of minor ions. These equations are presented in Section 2. In the course of deriving the diffusion and heat flow equations, we have discovered some new transport terms, and in Section 3 we discuss their possible importance to ionospheric studies. In Section 4 we present a summary, which includes references to the formulae that are relevant to ionospheric modeling.

\section{THEORETICAL FORMULATION}

Schunk (1975) has presented a general system of transport equations for the Earth's upper atmosphere and ionosphere. This system of equations contains a continuity, momentum, internal energy, stress tensor and heat flow equation for each species in the gas mixture. In this section of the paper, the general system of transport equations is applied to the mid-latitude topside ionosphere. The restriction to this region of the ionosphere enables us to make several simplifying assumptions which significantly reduce the general system of transport equations. First, we consider a fully-ionized plasma composed of two major ions, electrons and a number of minor ions. Next, we assume a steady state and take account of the fact that the species temperature and flow velocity differences are small in the mid-latitude topside ionosphere. This enables us to neglect stress and nonlinear acceleration terms and use Burgers' linear collision terms (Burgers, 1969). In addition, we neglect density and temperature gradients perpendicular to geomagnetic field lines. With this latter assumption, we need only consider plasma transport along geomagnetic field lines.

The derivation of the diffusion and heat flow equations follows from Schunk's momentum (2.7b and 5.1b) and heat flow (2.16 and 5.1e) equations. With the above simplifying assumptions, these equations become

$$
\begin{aligned}
\nabla p_{s}-n_{s} m_{s} \mathbf{G}-n_{s} e_{s} \mathbf{E}= & -n_{s} m_{s} \sum_{t} \nu_{s t}\left(\mathbf{u}_{s}-\mathbf{u}_{t}\right) \\
& +\frac{3}{5} \sum_{t} \nu_{s t} \frac{\mu_{s t}}{k T_{s t}}\left[\mathbf{q}_{s}-\frac{\rho_{s}}{\rho_{t}} \mathbf{q}_{t}\right] \\
-\frac{5}{2} \frac{k p_{s}}{m_{s}} \nabla T_{s}= & \mathbf{q}_{s}\left\{\frac{4}{5} \nu_{s s}+\sum_{t \neq s} \nu_{s t}\left[D_{s t}^{(1)}+\frac{3}{2} \frac{\mu_{s t}}{m_{s}} \frac{T_{s}}{T_{s t}}\right]\right\} \\
& -\sum_{t \neq s} \nu_{s t}\left[D_{s t}^{(4)}+\frac{3}{2} \frac{\mu_{s t}}{m_{s}} \frac{T_{s}}{T_{s t}}\right] \frac{\rho_{s}}{\rho_{t}} \mathbf{q}_{t} \\
& -\frac{3}{2} p_{s} \sum_{t \neq s} \frac{m_{t} \nu_{s t}}{m_{s}+m_{t}}\left(\mathbf{u}_{s}-\mathbf{u}_{t}\right),
\end{aligned}
$$

where

$$
\begin{aligned}
\nu_{s t} & =\frac{16 \sqrt{\pi}}{3} \frac{n_{t} m_{t}}{m_{s}+m_{t}}\left(\frac{\mu_{s t}}{2 k T_{s t}}\right)^{3 / 2} \frac{e_{s}^{2} e_{t}^{2}}{\mu_{s t}^{2}} \ln \Lambda \\
D_{s t}^{(1)} & =\left(3 m_{s}^{2}+\frac{1}{10} m_{s} m_{t}-\frac{1}{5} m_{t}^{2}\right) /\left(m_{s}+m_{t}\right)^{2} \\
D_{s t}^{(4)} & =\left(\frac{6}{5} m_{t}^{2}-\frac{3}{2} m_{s} m_{t}\right) /\left(m_{s}+m_{t}\right)^{2} \\
T_{s t} & =\left(m_{t} T_{s}+m_{s} T_{t}\right) /\left(m_{s}+m_{t}\right) \\
\mu_{s t} & =m_{s} m_{t} /\left(m_{s}+m_{t}\right),
\end{aligned}
$$

and where we have used the momentum equation to simplify the heat flow equation. In equations (1-7), $n_{s}$ is the density of species $s, m_{s}$ is the mass, $e_{s}$ is the charge, $\rho_{s}=n_{s} m_{s}$ is the mass density, $T_{s}$ is the temperature, $p_{s}=n_{s} k T_{s}$ is the partial pressure, $u_{s}$ is the drift or flow velocity, $q_{s}$ is the heat flow vector, $\mathbf{G}$ is the acceleration due to gravity, $\mathbf{E}$ is the electric field, $k$ is Boltzmann's constant and $\ln \Lambda$ is the Coulomb logarithm (cf. Burgers, 1969; Schunk, 1975).

The ion and electron densities and flow velocities are not independent, but are coupled through the requirements of charge neutrality and charge conservation. In the steady state, these requirements 
become

$$
\sum_{s} n_{s} e_{s}=0
$$

and

$$
\sum_{s} n_{s} e_{s} \mathbf{u}_{s}=0
$$

provided no current flows parallel to the geomagnetic field.

\section{Equations for the major ions and electrons}

The momentum (1) and heat flow (2) equations can be applied separately for each major ion (subscripts $i$ and $j$ ) and for the electrons (subscript $e$ ). The resulting system of equations is coupled, since heat flow and drift velocity terms for all species appear in each momentum and heat flow equation. The presence of heat flow terms in the momentum equations accounts for thermal diffusion effects, while the presence of drift velocity terms in the heat flow equations accounts for thermoelectric effects.

To cast the momentum and heat flow equations in the classical form, it is convenient to first consider the heat flow equations. Substituting $s=i, j$ and $e$, respectively, in equation (2), we obtain three equations which can be solved to obtain explicit expressions for $\mathbf{q}_{i}, \mathbf{q}_{j}$ and $\mathbf{q}_{e}$ in terms of the species temperature gradients and flow velocity differences. Neglecting terms of order $\left(m_{e} / m_{i}\right)^{1 / 2},\left(m_{e} / m_{j}\right)^{1 / 2}$ and $\left(T_{s}-T_{t}\right) / T_{s}$, these expressions become

$$
\begin{aligned}
& \mathbf{q}_{i}=\frac{1}{1-\xi}\left[-\lambda_{i} \nabla T_{i}\right.-\frac{\rho_{i}}{\rho_{j}} \frac{\nu_{i j}{ }^{\prime}}{\nu_{i}^{\prime}} \lambda_{j} \nabla T_{i} \\
&\left.+\left(\delta_{i j}-\frac{\rho_{i}}{\rho_{j}} \frac{\nu_{i j}^{\prime}}{\nu_{i}^{\prime}} \delta_{j i}\right)\left(\mathbf{u}_{i}-\mathbf{u}_{j}\right)\right] \\
& \mathbf{q}_{j}=\frac{1}{1-\xi}\left[-\lambda_{j} \nabla T_{j}-\frac{\rho_{j}}{\rho_{i}} \frac{\nu_{j i}^{\prime}}{\nu_{j}^{\prime}} \lambda_{i} \nabla T_{i}\right. \\
&\left.\quad+\left(\delta_{i j}-\frac{\rho_{j}}{\rho_{i}} \frac{\nu_{j i}^{\prime}}{\nu_{j}^{\prime}} \delta_{i j}\right)\left(\mathbf{u}_{i}-\mathbf{u}_{i}\right)\right]
\end{aligned}
$$

where the thermal conductivities and thermoelectric coefficients are given by

$$
\begin{gathered}
\lambda_{s}=\frac{25}{8} \frac{k p_{s}}{m_{s} \nu_{s}^{\prime}} \\
\delta_{s t}=\frac{15}{8} \frac{\mu_{s t} \nu_{s t}}{m_{s} v_{s}^{\prime}} p_{s}
\end{gathered}
$$

for $s$ and $t$ equal to $i, j, e$ and where

$$
\xi=\nu_{i j}{ }^{\prime} \nu_{j i}{ }^{\prime} /\left(\nu_{i}^{\prime} \nu_{j}^{\prime}\right) \text {. }
$$

In equations (10)-(15), the quantities $\nu_{e}{ }^{\prime}, \nu_{i}{ }^{\prime}, \nu_{j}{ }^{\prime}, \nu_{i j}{ }^{\prime}$ and $\nu_{j i}{ }^{\prime}$ are effective collision frequencies; the expressions are given in the Appendix.

Several important features should be noted about the heat flow expressions. First, there is a strong coupling between the ion gases in the sense that a temperature gradient in one gas causes heat to flow in both gases. Also, a relative drift between the ion gases causes heat to flow in both gases. On the other hand, a temperature gradient in the electron gas or a relative flow between the electron and ion gases has no effect on the ion gases. This latter result stems from our neglect of terms of order $\left(m_{e} / m_{i}\right)^{1 / 2},\left(m_{e} / m_{i}\right)^{1 / 2}$ and $\left(T_{s}-T_{t}\right) / T_{s}$. The neglect of similar terms in the electron heat flow equation accounts for the absence of ion temperature gradient terms in the expression for the electron heat flow. Consequently, for $\mathbf{q}_{\mathbf{e}}$ we obtain the familiar result that electron heat flows are caused by electron temperature gradients and relative electronion drifts (thermoelectric effect).

With the aid of the heat flow equations (10)-(12), it is now possible to derive diffusion equations of the classical form for the major ions and electrons. Since electrons are more mobile than ions, the electrons establish the polarization electrostatic field. Setting $s=e$ in equation (1) and eliminating the heat flow terms with the aid of equations (10)(12), we obtain

$$
\begin{aligned}
& e \mathbf{E}=-\frac{1}{n_{e}} \nabla p_{e}-\frac{15}{8} \frac{\nu_{e i}+\nu_{e j}}{\nu_{e}{ }^{\prime}} k \nabla T_{e} \\
& -m_{e}\left[1-\frac{9}{8} \frac{\nu_{e i}+\nu_{e i}}{\nu_{e}^{\prime}}\right]\left[\nu_{e i}\left(\mathbf{u}_{e}-\mathbf{u}_{i}\right)+\nu_{e i}\left(\mathbf{u}_{e}-\mathbf{u}_{i}\right)\right],
\end{aligned}
$$

where we have neglected terms of order $m_{e} / m_{i}$ and $m_{e} / m_{j}$ compared to terms of order one. When equation (16) is substituted into the ion momentum equations, only the first two terms on the righthand side survive, since the relative flow terms are of order $\left(m_{e} / m_{i}\right)^{1 / 2}$ and $\left(m_{e} / m_{i}\right)^{1 / 2}$ or smaller in comparison with other terms and must be neglected to be consistent with previous assumptions. Therefore, effectively the polarization electrostatic field becomes

$$
e \mathbf{E}=-\frac{1}{n_{e}} \nabla p_{e}-\frac{\frac{15 \sqrt{2}}{8}\left(n_{i} z_{i}^{2}+n_{j} z_{j}^{2}\right)}{n_{i} z_{i}+n_{j} z_{j}+\frac{13 \sqrt{2}}{8}\left(n_{i} z_{i}^{2}+n_{j} z_{j}^{2}\right)} k \nabla T_{e},
$$

where we have used the collision frequency expression (3) and the equation for charge neutrality (8) 
and where $z_{i}$ and $z_{i}$ are the ion charge numbers. Equation (17) agrees with the result obtained by Schunk and Walker (1969) for a topside ionosphere in diffusive equilibrium. The second term on the right-hand side of equation (17) represents the influence of thermal diffusion and can be traced to the effect of electron heat flow on electron momentum transport.

The ion diffusion equations are obtained by setting $s=i$ and $s=j$, respectively, in equation (1), by using equation (16) or (17) for the polarization electrostatic field, and by using equations (10)-(12) for the ion and electron heat flow vectors. Neglecting terms of order $\left(m_{e} / m_{i}\right)^{1 / 2}$ and $\left(m_{\varepsilon} / m_{j}\right)^{1 / 2}$ and solving for the ion flow velocities, these diffusion equations become

$$
\begin{aligned}
\mathbf{u}_{i}= & \mathbf{u}_{i}-D_{i}\left[\frac{1}{n_{i}} \nabla n_{i}-\frac{m_{i} \mathbf{G}}{k T_{i}}+\frac{1}{T_{i}} \nabla T_{i}+z_{i} \frac{T_{j} / T_{i}}{n_{e}} \nabla n_{e}\right. \\
& \left.+\frac{\left(z_{i}-\gamma_{i}\right)}{T_{i}} \nabla T_{e}+\frac{n_{i}}{n_{i}+n_{j}}\left(\frac{\alpha_{i j}}{T_{i}} \nabla T_{i}-\frac{\alpha_{i j}^{*}}{T_{i}} \nabla T_{i}\right)\right] \\
\mathbf{u}_{j}= & \mathbf{u}_{i}-D_{j}\left[\frac{1}{n_{j}} \nabla n_{j}-\frac{m_{j} \mathbf{G}}{k T_{j}}+\frac{1}{T_{i}} \nabla T_{i}+z_{j} \frac{T_{e} / T_{j}}{n_{e}} \nabla n_{e}\right. \\
& \left.+\frac{\left(z_{i}+\gamma_{i}\right)}{T_{i}} \nabla T_{e}-\frac{n_{i}}{n_{i}+n_{j}}\left(\frac{\alpha_{i j}}{T_{j}} \nabla T_{i}-\frac{\alpha_{i j}{ }^{*}}{T_{j}} \nabla T_{i}\right)\right],
\end{aligned}
$$

where the ordinary $\left(D_{i}, D_{j}\right)$ and thermal $\left(\alpha_{i j}\right.$, $\left.\alpha_{i j}{ }^{*}, \gamma_{i}, \gamma_{j}\right)$ diffusion coefficients are given by

$$
\begin{aligned}
D_{i} & =\frac{k T_{i}}{m_{i} \nu_{i j}} \frac{1}{\left(1-\Delta_{i j}\right)} \\
D_{i} & =\frac{k T_{i}}{m_{j} \nu_{i i}} \frac{1}{\left(1-\Delta_{i j}\right)} \\
\gamma_{i} & =\frac{15\left[\frac{n_{e}}{n_{i}} \nu_{e i}-z_{i}\left(\nu_{e i}+\nu_{e j}\right)\right]}{13\left(\nu_{e i}+\nu_{e j}\right)+8 \nu_{e e}} \\
\gamma_{j} & =\frac{15\left[z_{j}\left(\nu_{e i}+\nu_{e j}\right)-\frac{n_{e}}{n_{j}} \nu_{e j}\right]}{13\left(\nu_{e i}+\nu_{e j}\right)+8 \nu_{e e}} \\
\alpha_{i j} & =\frac{15}{8} \frac{\left(n_{i}+n_{j}\right)}{n_{i}} \frac{\mu_{i j}}{m_{i}} \frac{T_{i}}{T_{i j}} \frac{\nu_{i j}\left(\nu_{j}^{\prime}-\nu_{i i}{ }^{\prime}\right)}{\left(\nu_{i j}^{\prime}-\nu_{i j} \nu_{j i}{ }^{\prime}\right)} \\
\alpha_{i j}^{*} & =\left(\frac{m_{i}}{m_{j}}\right)^{2}\left(\frac{T_{i}}{T_{i}}\right) \frac{\left(\nu_{i}^{\prime}-\nu_{i j}{ }^{\prime}\right)}{\left(\nu_{i}^{\prime}-\nu_{i j}{ }^{\prime}\right)} \alpha_{i j},
\end{aligned}
$$

where

$$
\Delta_{i j}=\frac{3}{5} \frac{n_{i}}{\left(n_{i}+n_{i}\right)} \frac{\mu_{i j}}{m_{i}}\left[\alpha_{i j}+\frac{\rho_{i}}{\rho_{i}} \alpha_{i j}^{*}\right] .
$$

Equations (18) and (19) indicate that a temperature gradient in either ion gas causes thermal diffusion in both gases. The corresponding ion thermal diffusion coefficients $\alpha_{i j}$ and $\alpha_{i j}{ }^{*}$ arise as a result of ion-ion collisions, and these coefficients depend only on the major ion densities, masses, charges and temperatures. Likewise, the ion ordinary diffusion coefficients $D_{j}$ and $D_{j}$ depend only on the major ion parameters.

However, electrons do have an effect upon the thermal diffusion coefficients $\gamma_{i}$ and $\gamma_{i}$. These coefficients can be expressed in terms of the ion and electron parameters by using the electron collision frequencies given in the Appendix. With a slight rearrangement of terms, we obtain

$$
\begin{aligned}
& \gamma_{i}=\frac{15 \sqrt{2}}{8} \frac{n_{i} z_{i} z_{j}\left(z_{i}-z_{j}\right)}{\frac{13 \sqrt{2}}{8}\left(n_{i} z_{i}^{2}+n_{i} z_{i}^{2}\right)+n_{i} z_{i}+n_{i} z_{j}} \\
& \gamma_{j}=\frac{15 \sqrt{2}}{8} \frac{n_{i} z_{i} z_{j}\left(z_{i}-z_{j}\right)}{\frac{13 \sqrt{2}}{8}\left(n_{i} z_{i}^{2}+n_{i} z_{i}^{2}\right)+n_{i} z_{i}+n_{i} z_{j}}
\end{aligned}
$$

where use has been made of the charge neutrality equation (8). Equations (27) and (28) agree with the corresponding thermal diffusion coefficients calculated by Schunk and Walker (1969). A complete discussion of these coefficients has been given by these authors, and we only note here that when the major ions have the same charge both $\gamma_{i}$ and $\gamma_{j}$ are zero.

Finally, we note that when the relative drift between the major ions is neglected and when the major ions are assumed to have a common temperature, our ion diffusion equations (18) and (19) reduce to the diffusive equilibrium equations of Schunk and Walker (1969), with $\left(\alpha_{i j}-\alpha_{i j}{ }^{*}\right)$ agreeing with their major ion thermal diffusion coefficient.

\section{Equations for a minor ion}

Diffusion and heat flow equations for a minor ion in a gas mixture composed of two major ions and electrons can be obtained from equations (1) and (2). Letting subscript $x$ correspond to the minor ion, the condition that an ion be minor is

$$
\frac{n_{x}}{n_{e}} \ll 1
$$

By virtue of equation (29), the equations for the major ions and electrons are unaffected by the presence of the minor ion.

To obtain the minor ion heat flow equation, we set $s=x$ in equation (2) and solve for $q_{x}$. If, as 
before, we neglect terms of order $\left(m_{e} / m_{s}\right)^{1 / 2}$, where $s=i, j$ or $x$ and assume small minor ion-major ion temperature differences, the minor ion heat flow equation becomes

$$
\begin{aligned}
\mathbf{q}_{x}=-\lambda_{x} \nabla T_{x}+\frac{\rho_{x}}{\rho_{i}} \frac{\nu_{x i} i^{\prime}}{\nu_{x}{ }^{\prime}} \mathbf{q}_{i}+\frac{\rho_{x}}{\rho_{i}} \frac{\nu_{x i}{ }^{\prime}}{\nu_{x}{ }^{\prime}} \mathbf{q}_{j} \\
+\delta_{x i}\left(\mathbf{u}_{x}-\mathbf{n}_{i}\right)+\delta_{x j}\left(\mathbf{u}_{x}-\mathbf{u}_{i}\right),
\end{aligned}
$$

where

$$
\begin{aligned}
& \lambda_{x}=\frac{25}{8} \frac{k p_{x}}{m_{x} \nu_{x}{ }^{\prime}} \\
& \delta_{x i}=\frac{15}{8} \frac{\mu_{x i} \nu_{x i}}{m_{x} \nu_{x}{ }^{\prime}} p_{x} \\
& \delta_{x i}=\frac{15}{8} \frac{\mu_{x i} \nu_{x j}}{m_{x} \nu_{x}{ }^{\prime}} p_{x},
\end{aligned}
$$

and where the major ion heat flow vectors $q_{i}$ and $q_{j}$ are given by equations (10) and (11). The quantities $\nu_{x}{ }^{\prime}, \nu_{x i}{ }^{\prime}$ and $\nu_{x j}{ }^{\prime}$ are effective collision frequencies and are given in the Appendix.

Equation (30) indicates that a temperature gradient in either of the major ion gases or in the minor ion gas causes heat to flow through the minor ion gas. Also, a relative drift between the major ions or between the minor ion and either major ion causes a minor ion heat flow.

To obtain the minor ion diffusion equation, we set $s=x$ in equation (1) and repeat the algebraic manipulations which led to the major ion diffusion equations. If we again assume small temperature differences and take account of the small electron mass, we obtain

$$
\begin{aligned}
\mathbf{u}_{x}= & h_{x i} \mathbf{u}_{i}+h_{x j} \mathbf{u}_{i} \\
& -D_{x}\left[\frac{1}{n_{x}} \nabla n_{x}-\frac{m_{x} \mathbf{G}}{k T_{x}}+\frac{1}{T_{x}} \nabla T_{x}+z_{x} \frac{T_{e} / T_{x}}{n_{e}} \nabla n_{e}\right. \\
& \left.+\frac{\left(z_{x}-\gamma_{x}\right)}{T_{x}} \nabla T_{e}-\frac{1}{T_{x}}\left(\beta_{x} \nabla T_{x}+\beta_{i} \nabla T_{i}+\beta_{i} \nabla T_{i}\right)\right],
\end{aligned}
$$

where the ordinary $\left(D_{x}\right)$ and thermal $\left(\beta_{x}, \beta_{i}, \beta_{i}, \gamma_{x}\right)$ diffusion coefficients are given by

$$
\begin{aligned}
& D_{x}=\frac{k T_{x} / m_{x}}{\nu_{x i}\left(1-\Delta_{x i}\right)+\nu_{x j}\left(1-\Delta_{x j}\right)} \\
& \gamma_{x}=\frac{15\left[\frac{n_{e}}{n_{x}} \nu_{e x}-z_{x}\left(\nu_{e i}+\nu_{e j}\right)\right]}{13\left(\nu_{e i}+\nu_{e j}\right)+8 \nu_{e e}} \\
& \beta_{s}=\frac{15}{8} \frac{m_{x} / m_{s}}{1-\xi} \sum_{t=i, j} \frac{\mu_{x t}}{m_{s}} \frac{\nu_{x t}}{\nu_{s}} \frac{T_{s}}{T_{x t}} Y_{s t}
\end{aligned}
$$

where $s=i, j$ or $x$ in the last expression and where

$$
\begin{aligned}
h_{x i} & =\frac{\nu_{x i}\left(1-\Delta_{x i}\right)-R_{i j}+R_{j i}}{\nu_{x i}\left(1-\Delta_{x i}\right)+\nu_{x i}\left(1-\Delta_{x j}\right)} \\
\Delta_{x i} & =\frac{9}{8} \frac{\mu_{x i}}{m_{x}} \sum_{t=i, j} \frac{\mu_{x t}}{m_{x}} \frac{\nu_{x t}}{\nu_{x}} \frac{T_{x}}{T_{x t}} \\
R_{i j} & =\frac{9}{8} \frac{\mu_{i j}}{m_{i}^{2}} \frac{\nu_{i j}}{\nu_{i}} \frac{T_{i}}{1-\xi}\left[\frac{\mu_{x i} \nu_{x i} Q_{i i}}{T_{x i}}+\frac{\mu_{x j} \nu_{x i} Q_{i j}}{T_{x j}}\right],
\end{aligned}
$$

where $\xi$ is defined by equation (15).

The quantities $h_{x j}, \Delta_{x j}$ and $R_{f j}$ can be obtained from the last three equations by interchanging the subscripts $i$ and $j$. The $Q$ 's and Y's are dimensionless quantities which depend on the various collision frequencies and are given in the Appendix.

Equation (34) indicates that the motion of the minor ion is influenced by the motion of both major ions in addition to ordinary and thermal diffusion processes. With regard to diffusion processes, it should be noted that a temperature gradient in either major ion gas or in the minor ion gas causes thermal diffusion in the minor ion gas. The corresponding thermal diffusion coefficients $\beta_{i}, \beta_{i}$ and $\beta_{x}$ arise solely from collisions between the various ion species. Likewise, the ordinary minor ion diffusion coefficient $D_{x}$ results from ion-ion interactions.

Electron-ion and electron-electron interactions influence the thermal diffusion coefficient $\gamma_{\boldsymbol{x}}$. Using the electron collision frequencies given in the Appendix, this coefficient can be expressed in the form

$$
\gamma_{x}=\frac{15 \sqrt{2}}{8} \frac{z_{x}^{2}\left(n_{i} z_{i}+n_{i} z_{j}\right)-z_{x}\left(n_{i} z_{i}^{2}+n_{j} z_{i}^{2}\right)}{\frac{13 \sqrt{2}}{8}\left(n_{i} z_{i}^{2}+n_{j} z_{j}^{2}\right)+n_{i} z_{i}+n_{j} z_{j}},
$$

where use has been made of the equation for charge neutrality (8). Equation (41) agrees with the corresponding thermal diffusion coefficient calculated by Schunk and Walker (1969). As with the major ion coefficients $\gamma_{i}$ and $\gamma_{j}, \gamma_{x}$ is zero when all of the ions have the same charge.

Finally, we note that when the major ion drift velocities $\mathbf{u}_{i}$ and $\mathbf{u}_{i}$ are set equal to zero and all the ions are assumed to have a common temperature, our minor ion diffusion equation (34) reduces to the diffusive equilibrium equation given by Schunk and Walker (1969), with $\left(\beta_{x}+\beta_{i}+\beta_{j}\right)$ agreeing with their minor ion thermal diffusion coefficient $\beta$.

\section{Comparison with Chapman and Cowling and others}

The 13-moment system of transport equations yields transport coefficients that are equivalent to 
those obtained by Chapman and Cowling (1970) if two terms in the Sonine polynomial expansion are retained in the latter formulation. Consequently, our ordinary diffusion coefficients correspond to what Chapman and Cowling call the "second approximation" to these coefficients, while our thermal diffusion coefficients and thermal conductivities correspond to the "first approximation".

As noted by Schunk and Walker (1970b), the second approximation to the ordinary diffusion coefficients can be greater than the first approximation values by as much as a factor of two for light minor ions such as $\mathrm{He}^{+}$and $\mathrm{H}^{+}$. In Table 1 , we present the ratio of the second to first approximation to the minor ion diffusion coefficient for different minor ions in an electron- $\mathrm{O}^{+}$plasma. The first approximation values are obtained from our diffusion coefficients by setting the $\Delta$ 's equal to zero. Also shown in Table 1 are the corresponding ratios obtained by Schunk and Walker (1970b) from the multicomponent formulation of Hirschfelder et al. (1964). As noted previously (Schunk and Walker, 1970a), the two formulations give comparable but not identical results.

From the comparison given in Table 1 and the work of Meador and Staton (1965) and Devoto (1966), it appears that our ordinary and thermal diffusion coefficients have an accuracy of about $20-30 \%$.

An estimate of the accuracy of our ion thermal conductivities can be obtained from a simple model considered by Chapman and Cowling (1970). For a simple gas composed of ions of equal mass and charge, half positive and half negative, Chapman and Cowling found the second approximation to the ion thermal conductivity to be a factor of 1.25 greater than the first approximation. Therefore, our ion thermal conductivities, which correspond to the first approximation, probably have the same accuracy as our ordinary and thermal diffusion coefficients, i.e. $20-30 \%$.

Of all the transport coefficients, the electron thermal conductivity is the least accurate. More

TABLE 1. RATIO OF THE SECOND AND FIRST APPROXIMATIONS TO THE MINOR ION DIFFUSION COEFFICIENT

\begin{tabular}{lcc}
\hline Minor ion & $\begin{array}{c}D_{x}(2) / D_{x}(1) \\
(13 \text {-noment) }\end{array}$ & $\begin{array}{c}D_{x}(2) / D_{x}(1) \\
\text { (Hirschfelder et al) }\end{array}$ \\
\hline $\mathrm{H}^{+}$ & 2.76 & 2.15 \\
$\mathrm{He}^{+}$ & 1.91 & 1.70 \\
$\mathrm{~N}^{+}$ & 1.22 & 1.18 \\
\hline
\end{tabular}

rigorous calculations of this coefficient by Spitzer and Härm (1953) and others indicate that the first approximation to the electron thermal conductivity is in error by about a factor of two. However, the electron gas effectively decouples from the ion gases and, therefore, electron transport coefficients can be calculated separately using, for example, the method of Shkarofsky (1961) (cf. Schunk and Walker, 1970c; Schunk, 1975).

\section{APPLICATION TO THE IONOSPHERE}

The diffusion and heat flow equations that we have presented in the previous section are more complex than transport equations previously used in mid-latitude ionospheric modeling studies. It is therefore instructive to study some of the transport coefficients in more detail.

Illustrative values of the major ion thermal diffusion coefficients $\alpha_{i j}$ and $\alpha_{i j}{ }^{*}$ are shown in Tables 2 and 3 for major ion mass ratios appropriate to ionospheric mixtures of ionized oxygen, nitrogen, helium and hydrogen. For completeness, we also present values for very heavy and very light ions. In generating these and subsequent tables, we have assumed that the various ions have a common temperature. The effect of different ion temperatures on the individual transport coefficients is discussed separately.

Tables 2 and 3 indicate that for $m_{i} / m_{j}>7 / 2, \alpha_{i j}{ }^{*}$ is much greater than $\alpha_{i j}$ and nearly equal to the negative of the major ion thermal diffusion coefficient, $\alpha_{s w}$, of Schunk and Walker. This implies that when there is a substantial mass difference between the major ions, the thermal diffusion of both the light and heavy ion is governed by the light ion temperature gradient [see equations (18) and (19)]. Since the $\mathrm{H}^{+}$temperature gradient is typically greater than the $\mathrm{O}^{+}$temperature gradient in the topside ionosphere, thermal diffusion effects are even more important than previously thought. For comparable major ion masses, thermal diffusion has a negligibly small effect on the ion density profiles.

With regard to the effect of the major ion temperature ratio $T_{i} / T_{j}$ on $\alpha_{i j}$ and $\alpha_{i j}{ }^{*}$, we note that these coefficients are sensitive to $T_{i} / T_{j}$ only when $m_{i} \approx m_{i}$. Therefore, for ionospheric mixtures of $\mathrm{H}^{+}-$ $\mathrm{O}^{+}, \mathrm{H}^{+}-\mathrm{He}^{+}$or $\mathrm{He}^{+}-\mathrm{O}^{+}$little error is introduced by setting $T_{i}=T_{j}$ in the expressions for $\alpha_{i j}$ and $\alpha_{i j}{ }^{*}$. We also note that when $m_{i} \rightarrow m_{j}$ and $T_{i}=T_{i}$ thermal diffusion does not occur, since the quantity $\left(\alpha_{i j}-\alpha_{i j}{ }^{*}\right) \rightarrow 0$. However, when $m_{i}=m_{j}$ and $T_{i} \neq T_{j}$, thermal diffusion persists. This latter effect appears to be a new result.

Although doubly-charged ions are not likely to 
TABle 2. Values of $\alpha_{i j}$ AS A function of $n_{i} / n_{i}$ AND $m_{i} / m_{i}$ FOR $z_{i} / z_{j}=1$ AND $T_{i} / T_{i}=1$

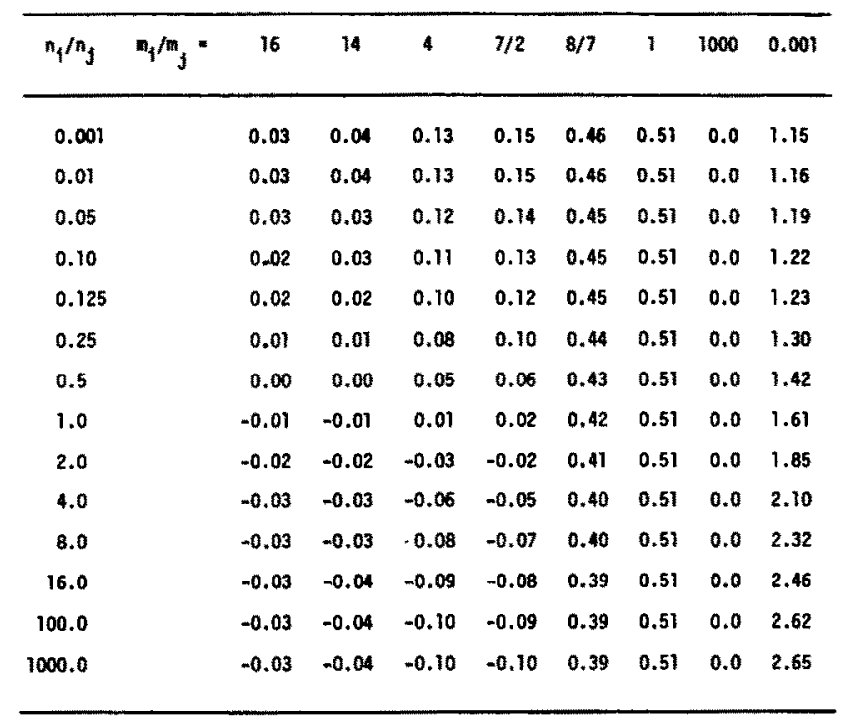

be major in the terrestrial ionosphere, we have studied the variation of $\alpha_{i j}$ and $\alpha_{i j}{ }^{*}$ with $n_{l} / n_{i j}, m_{i} / m_{i}$ and $T_{i} / T_{i}$ for $z_{i} / z_{i}=2$, and we found the same qualitative behaviour as for $z_{i} / z_{j}=1$.

In Table 4, we present illustrative values of the correction factor, $\Delta_{i j}$, which appears in the major ion diffusion coefficients. This quantity, which accounts for the effect of heat flow on ordinary diffusion, attains its greatest value in the limit when one of the major ions becomes a very light minor ion. In this limit, $\Delta_{i i}$ assumes its maximum value of 0.69 , and this results in a factor of 3.25 increase in both the major and minor ion diffusion coefficients. For comparison, $\Delta_{i j}$ acts to increase the $\mathrm{H}^{+}$and $\mathrm{O}^{+}$ diffusion coefficients by a factor of 2.76 when $\mathrm{H}^{+}$ becomes a minor ion (see Table 1). In the opposite limit of a very heavy minor ion, $\Delta_{i j}$ is zero. However, as the density of the heavy minor ion increases, $\Delta_{i j}$ rapidly becomes important. For an $\mathrm{H}^{+}$ $\mathrm{O}^{+}$mixture with $\mathrm{O}^{+}$minor, $\Delta_{i j}$ attains half of its

TABLE 3. VALUES OF $\alpha_{i j}$ AS A FUnCTION of $n_{j} / n_{i}$ AND $m_{i} / m_{i}$ FOR $z_{i} / z_{i}=1$ AND $T_{i} / T_{1}=1$

\begin{tabular}{|c|c|c|c|c|c|c|c|c|c|}
\hline$n_{i} / n_{j}$ & $m_{i} / m_{j}=$ & 16 & 14 & 4 & $7 / 2$ & $8 / 7$ & 1 & 1000 & 0.001 \\
\hline 0.001 & & 2.41 & 2.38 & 1.80 & 1.71 & 0.64 & 0.51 & 2.65 & 0.0 \\
\hline 0.01 & & $2+39$ & 2.35 & 1.79 & 1.69 & 0.64 & 0.51 & 2.62 & 0.0 \\
\hline 0.05 & & 2.29 & 2.26 & 1.74 & 1.65 & 0.63 & 0.51 & 2.50 & 0.0 \\
\hline 0.10 & & 2.19 & 2.16 & 1.69 & 1.60 & 0.63 & 0.51 & 2.37 & 0.0 \\
\hline 0.125 & & 2.14 & 2.12 & 1.67 & 1.58 & 0.63 & 0.51 & 2.32 & 0.0 \\
\hline 0.25 & & 1.97 & 1.95 & 1.57 & 1.50 & 0.62 & 0.51 & 2.10 & 0.0 \\
\hline 0.5 & & 1.75 & 1.74 & 1.44 & 1.38 & 0.61 & 0.51 & 1.85 & 0.0 \\
\hline 1.0 & & 1.54 & 1.53 & 1.30 & 1.25 & 0.60 & 0.51 & 1.61 & 0.0 \\
\hline 2.0 & & 1.37 & 1.37 & 1.18 & 1.14 & 0.59 & 0.51 & 1.42 & 0.0 \\
\hline 4.0 & & 1.26 & 1.26 & 1.10 & 1.07 & 0.58 & 0.51 & 1.30 & 0,0 \\
\hline 8.0 & & 1.20 & 1.20 & 1.05 & 1.02 & 0.57 & 0.51 & 1.23 & 0.0 \\
\hline 16.0 & & 1.17 & 1.16 & 1.02 & 0.99 & 0.57 & 0.51 & 1.19 & 0.0 \\
\hline 100.0 & & 1.13 & 1.13 & 1.00 & 0.97 & 0.56 & 0.51 & 1.16 & 0.0 \\
\hline 1000.0 & & 1.13 & 1.12 & 0.99 & 0.96 & 0.56 & 0.51 & 1.15 & 0.0 \\
\hline
\end{tabular}


TABle 4. Values of $\Delta_{i j}$ AS A FUNCTION OF $n_{i} / n_{j}$ AND $m_{i} / m_{j}$ fOR $z_{i} / z_{j}=1$ AND $T_{i} / T_{j}=1$

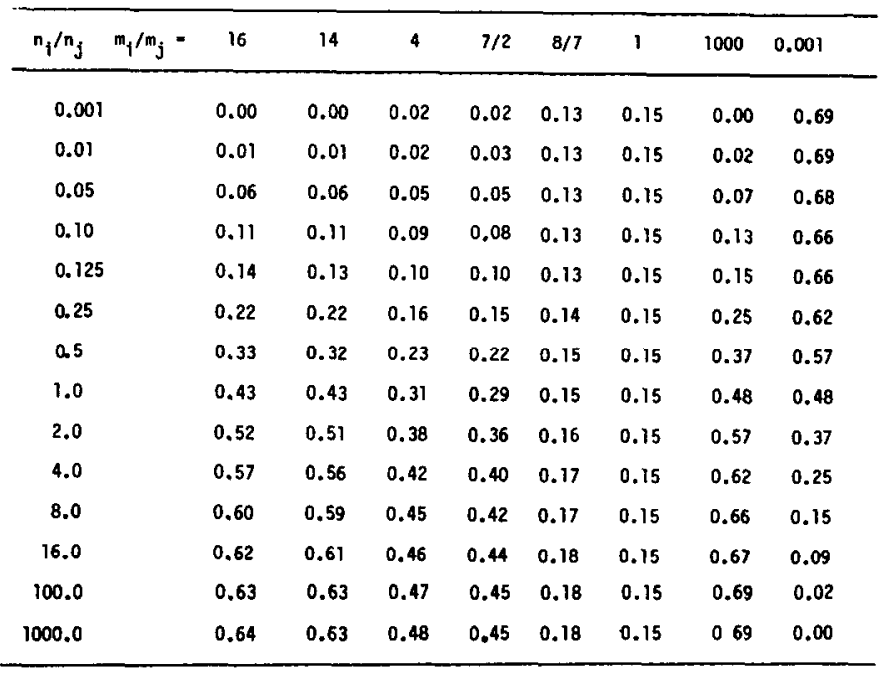

maximum value when the $\mathrm{O}^{+}$concentration reaches about $33 \%$.

We have found that $\Delta_{i j}$ is not sensitive to $T_{i} / T_{i}$, and little error is introduced by setting $T_{i}=T_{j}$ in the expression for $\Delta_{i j}$.

Illustrative values of the minor ion thermal diffusion coefficients $\beta_{x}, \beta_{i}$ and $\beta_{i}$ are given in Tables 5-7 for various minor ions in mixtures of $\mathrm{O}^{+}$(subscript $i$ ) and $\mathrm{H}^{+}$(subscript $j$ ). Also shown in Tables 5-7 are values for the limiting eases of a very light (Lorentz limit) and a very heavy (Rayleigh limit) minor ion. These tables exhibit several important features. First, it should be noted that $\beta_{x}$ generally has a sign that is opposite to that of $\beta\left(\mathrm{O}^{+}\right)$and $\beta\left(\mathrm{H}^{+}\right)$. Consequently, the thermal diffusion force due to the minor ion temperature gradient generally opposes the thermal diffusion forces due to the major ion temperature gradients. Also, when one of the major ions becomes a minor ion, its associated thermal diffusion coefficient becomes small, with the consequent result that its temperature gradient no longer affects the other minor ions.

It should also be noted that thermal diffusion has

Table 5. Values of $\beta_{x}$ for various ions in mixtures of $\mathrm{O}^{+}$AND $\mathrm{H}^{+}$AND FOR $T_{x}=T\left(O^{+}\right)=T\left(H^{+}\right)$

\begin{tabular}{ccccccc}
\hline$n\left(0^{+}\right) / n^{+}\left(\mathrm{H}^{+}\right)$ & $\mathrm{He}^{+}$ & $\mathrm{N}^{+}$ & $\mathrm{O}^{++}$ & $\mathrm{N}^{++}$ & Rayleigh & Lorentz \\
\hline 0.001 & -0.14 & -0.04 & -0.03 & -0.04 & 0.0 & -1.15 \\
0.01 & -0.14 & -0.04 & -0.04 & -0.04 & 0.0 & -1.15 \\
0.05 & -0.18 & -0.07 & -0.07 & -0.07 & 0.0 & -1.15 \\
0.10 & -0.22 & -0.11 & -0.10 & -0.11 & 0.0 & -1.15 \\
0.125 & -0.24 & -0.12 & -0.11 & -0.12 & 0.0 & -1.15 \\
0.25 & -0.32 & -0.18 & -0.17 & -0.18 & 0.0 & -1.15 \\
0.5 & -0.44 & -0.26 & -0.24 & -0.26 & 0.0 & -1.15 \\
1.0 & -0.58 & -0.35 & -0.32 & -0.35 & 0.0 & -1.15 \\
2.0 & -0.72 & -0.43 & -0.39 & -0.43 & 0.0 & -1.15 \\
4.0 & -0.83 & -0.49 & -0.44 & -0.49 & 0.0 & -1.15 \\
8.0 & -0.91 & -0.52 & -0.47 & -0.52 & 0.0 & -1.15 \\
16.0 & -0.95 & -0.54 & -0.49 & -0.54 & 0.0 & -1.15 \\
100.0 & -0.99 & -0.56 & -0.51 & -0.56 & 0.0 & -1.15 \\
1000.0 & -0.99 & -0.56 & -0.51 & -0.56 & 0.0 & -1.15 \\
\hline
\end{tabular}


Table 6. Values of $\beta\left(\mathrm{O}^{+}\right)$fOR Various IONS IN MIXTURES OF ${\mathrm{O}^{+}}^{+}$AND $\mathrm{H}^{+}$AND For $T_{x}=T\left(O^{+}\right)=T\left(H^{+}\right)$

\begin{tabular}{ccccccc}
\hline$n\left(0^{+}\right) / n\left(\mathrm{H}^{+}\right)$ & $\mathrm{He}^{+}$ & $\mathrm{N}^{+}$ & $\mathrm{O}^{++}$ & $\mathrm{N}^{++}$ & Rayleigh & Lorentz \\
\hline 0.001 & 0.00 & 0.00 & 0.00 & 0.00 & 0.00 & 0.0 \\
0.01 & 0.00 & 0.01 & 0.04 & 0.03 & 0.02 & 0.0 \\
0.05 & 0.01 & 0.04 & 0.17 & 0.15 & 0.11 & 0.0 \\
0.10 & 0.02 & 0.07 & 0.31 & 0.28 & 0.22 & 0.0 \\
0.125 & 0.02 & 0.08 & 0.37 & 0.33 & 0.27 & 0.0 \\
0.25 & 0.04 & 0.14 & 0.63 & 0.56 & 0.48 & 0.0 \\
0.5 & 0.04 & 0.21 & 0.96 & 0.84 & 0.81 & 0.0 \\
1.0 & 0.03 & 0.28 & 1.31 & 1.12 & 1.24 & 0.0 \\
2.0 & 0.01 & 0.33 & 1.60 & 1.32 & 1.69 & 0.0 \\
4.0 & -0.03 & 0.36 & 1.79 & 1.44 & 2.06 & 0.0 \\
8.0 & -0.06 & 0.38 & 1.91 & 1.50 & 2.32 & 0.0 \\
16.0 & -0.08 & 0.38 & 1.97 & 1.53 & 2.47 & 0.0 \\
100.0 & -0.10 & 0.39 & 2.02 & 1.55 & 2.62 & 0.0 \\
1000.0 & -0.10 & 0.39 & 2.03 & 1.55 & 2.64 & 0.0 \\
\hline
\end{tabular}

a greater effect on the minor ion when $\mathrm{H}^{+}$is the dominant major ion than when $\mathrm{O}^{+}$dominates. In addition, when $\mathrm{H}^{+}$dominates both $\beta_{x}$ and $\beta\left(\mathrm{O}^{+}\right)$ are much smaller than $\beta\left(\mathrm{H}^{+}\right)$, so that the $\mathrm{H}^{+}$temperature gradient establishes the thermal diffusion force on the minor ion. On the other hand, when $\mathrm{O}^{+}$dominates the thermal diffusion force may be established by either the $\mathrm{O}^{+}$temperature gradient or the minor ion temperature gradient depending on the mass and charge of the minor ion. The lighter the minor ion the more important is the minor ion temperature gradient.
In the Lorentz limit of a very light minor ion, only the minor ion temperature gradient is effective in establishing the thermal diffusion force on the minor ion. In the opposite limit of a very heavy minor ion, the thermal diffusion force on the minor ion is established by the $\mathrm{H}^{+}$temperature gradient if $\mathrm{H}^{+}$dominates and by the $\mathrm{O}^{+}$temperature gradient if $\mathrm{O}^{+}$dominates.

In general, the thermal diffusion coefficients $\beta_{x}$, $\beta_{i}$ and $\beta_{j}$ are all complex functions of $T_{x}, T_{i}$ and $T_{i}$. When $\mathrm{O}^{+}$is the dominant major ion, $\beta\left(\mathrm{O}^{+}\right)$is affected most by the temperature ratio $T_{x} / T\left(O^{+}\right)$.

TABle 7. Values of $\beta\left(H^{+}\right)$FOR VARIOUS IONS IN MIXTURes OF ${0^{+}}^{+}$AND $\mathbf{H}^{+}$AND FOR

\begin{tabular}{ccccccc}
\multicolumn{7}{c}{$T_{x}=T\left(\mathrm{O}^{+}\right)=T\left(\mathrm{H}^{+}\right)$} \\
\hline $\mathrm{n}\left(\mathrm{O}^{+}\right) / \mathrm{n}\left(\mathrm{H}^{+}\right)$ & $\mathrm{He}$ & $\mathrm{N}^{+}$ & $\mathrm{O}^{++}$ & $\mathrm{N}^{++}$ & Rayleigh & Lorentz \\
\hline 0.001 & 1.80 & 2.38 & 9.63 & 9.50 & 2.65 & 0.0 \\
0.01 & 1.76 & 2.33 & 9.45 & 9.32 & 2.60 & 0.0 \\
0.05 & 1.61 & 2.15 & 8.72 & 8.60 & 2.42 & 0.0 \\
0.10 & 1.44 & 1.96 & 7.96 & 7.84 & 2.23 & 0.0 \\
0.125 & 1.37 & 1.88 & 7.62 & 7.50 & 2.15 & 0.0 \\
0.25 & 1.10 & 1.55 & 6.30 & 6.19 & 1.81 & 0.0 \\
0.5 & 0.78 & 1.14 & 4.67 & 4.58 & 1.38 & 0.0 \\
1.0 & 0.48 & 0.75 & 3.08 & 3.00 & 0.94 & 0.0 \\
2.0 & 0.27 & 0.45 & 1.83 & 1.78 & 0.58 & 0.0 \\
4.0 & 0.14 & 0.25 & 1.01 & 0.98 & 0.33 & 0.0 \\
8.0 & 0.07 & 0.13 & 0.53 & 0.52 & 0.18 & 0.0 \\
16.0 & 0.04 & 0.07 & 0.27 & 0.26 & 0.09 & 0.0 \\
100.0 & 0.01 & 0.01 & 0.05 & 0.04 & 0.02 & 0.0 \\
1000.0 & 0.00 & 0.00 & 0.01 & 0.00 & 0.00 & 0.0 \\
\hline & & & & & & \\
\hline
\end{tabular}


Table 8. Values of $\Delta\left(x, \mathrm{O}^{+}\right)$For various ions In mixtures of $\mathrm{O}^{+}$and $\mathrm{H}^{+}$and for $T_{x}=T\left(O^{+}\right)=T\left(H^{+}\right)$

\begin{tabular}{ccccccc}
\hline$n\left(0^{+}\right) / \mathrm{n}\left(\mathrm{H}^{+}\right)$ & $\mathrm{He}^{+}$ & $\mathrm{N}^{+}$ & $0^{++}$ & $\mathrm{N}^{++}$ & Rayleigh & Lorentz \\
\hline 0.001 & 0.07 & 0.01 & 0.01 & 0.01 & 0.0 & 0.69 \\
0.01 & 0.07 & 0.61 & 0.01 & 0.01 & 0.0 & 0.69 \\
0.05 & 0.09 & 0.02 & 0.02 & 0.02 & 0.0 & 0.69 \\
0.10 & 0.11 & 0.03 & 0.03 & 0.03 & 0.0 & 0.69 \\
0.125 & 0.11 & 0.04 & 0.03 & 0.04 & 0.0 & 0.69 \\
0.25 & 0.15 & 0.06 & 0.05 & 0.06 & 0.0 & 0.69 \\
0.5 & 0.21 & 0.08 & 0.07 & 0.08 & 0.0 & 0.69 \\
1.0 & 0.28 & 0.11 & 0.10 & 0.11 & 0.0 & 0.69 \\
2.0 & 0.35 & 0.14 & 0.12 & 0.14 & 0.0 & 0.69 \\
4.0 & 0.40 & 0.16 & 0.13 & 0.16 & 0.0 & 0.69 \\
8.0 & 0.43 & 0.17 & 0.14 & 0.17 & 0.0 & 0.69 \\
16.0 & 0.45 & 0.17 & 0.15 & 0.17 & 0.0 & 0.69 \\
100.0 & 0.47 & 0.18 & 0.15 & 0.18 & 0.0 & 0.69 \\
1000.0 & 0.48 & 0.18 & 0.15 & 0.18 & 0.0 & 0.69 \\
& & & & & &
\end{tabular}

Likewise, when $\mathrm{H}^{+}$is the dominant major ion, $\beta\left(\mathrm{H}^{+}\right)$is most sensitive to $T_{x} / T\left(\mathrm{H}^{+}\right)$. As far as $\beta_{x}$ is concerned, $T_{x} / T\left(\mathrm{O}^{+}\right)$is the most important temperature ratio when $\mathrm{O}^{+}$dominates, while when $\mathrm{H}^{+}$ dominates, $T_{x} / T\left(\mathrm{H}^{+}\right)$is slightly more important than $T_{x} / T\left(\mathrm{O}^{+}\right)$. For comparable major ion densities, $\beta_{\mathbf{x}}$ is most sensitive to the temperature ratio $T\left(\mathrm{O}^{+}\right) / T\left(\mathrm{H}^{+}\right)$.

In Tables 8 and 9, we present illustrative values of the minor ion correction factors $\Delta_{x i}$ and $\Delta_{x j}$ for various minor ions in mixtures of $\mathrm{O}^{+}$(subscript $i$ ) and $\mathrm{H}^{+}$(subscript $j$ ). These correction factors appear in the minor ion diffusion (35) and frictional drag (38) coefficients. As with the major ion correction factor $\Delta_{i}$, the minor ion correction factors take on their maximum values in the limit of a very light minor ion. This tendency is clearly illustrated in Tables 8 and 9 by the increase in both $\Delta\left(x, O^{+}\right)$and $\Delta\left(x, \mathrm{H}^{+}\right)$with increasing $\mathrm{O}^{+}$concentration. It should also be noted that both $\Delta\left(x, \mathrm{O}^{+}\right)$and $\Delta\left(x, \mathrm{H}^{+}\right)$are independent of the charge of the minor ion (compare $\mathrm{N}^{+}$and $\mathrm{N}^{2+}$ columns).

Table 9. Values of $\Delta\left(x, H^{+}\right)$For various ions IN MDXTURes of $\mathrm{O}^{+}$and $\mathrm{H}^{+}$and For $T_{x}=$ $T\left(\mathrm{O}^{+}\right)=T\left(\mathrm{H}^{+}\right)$

\begin{tabular}{ccccccc}
\hline$n\left(0^{+}\right) / n\left(\mathrm{H}^{+}\right)$ & $\mathrm{He}^{+}$ & $\mathrm{N}^{+}$ & $\mathrm{O}^{++}$ & $\mathrm{N}^{++}$ & Rayleigh & Lorentz \\
\hline 0.001 & 0.02 & 0.00 & 0.00 & 0.00 & 0.0 & 0.69 \\
0.01 & 0.02 & 0.00 & 0.00 & 0.00 & 0.0 & 0.69 \\
0.05 & 0.02 & 0.00 & 0.00 & 0.00 & 0.0 & 0.69 \\
0.10 & 0.03 & 0.00 & 0.00 & 0.00 & 0.0 & 0.69 \\
0.125 & 0.03 & 0.01 & 0.00 & 0.01 & 0.0 & 0.69 \\
0.25 & 0.04 & 0.01 & 0.01 & 0.01 & 0.0 & 0.69 \\
0.5 & 0.05 & 0.01 & 0.01 & 0.01 & 0.0 & 0.69 \\
1.0 & 0.07 & 0.01 & 0.01 & 0.01 & 0.0 & 0.69 \\
2.0 & 0.09 & 0.02 & 0.01 & 0.02 & 0.0 & 0.69 \\
4.0 & 0.10 & 0.02 & 0.02 & 0.02 & 0.0 & 0.69 \\
8.0 & 0.11 & 0.02 & 0.02 & 0.02 & 0.0 & 0.69 \\
16.0 & 0.11 & 0.02 & 0.02 & 0.02 & 0.0 & 0.69 \\
100.0 & 0.12 & 0.02 & 0.02 & 0.02 & 0.0 & 0.69 \\
1000.0 & 0.12 & 0.02 & 0.02 & 0.02 & 0.0 & 0.69 \\
\hline
\end{tabular}


With regard to the temperature dependence of the minor ion correction factors, we note that temperature effects are important only when the $\mathrm{O}^{+} / \mathrm{H}^{+}$density ratio is smaller than about 0.1 . However, in this limit the correction factors are small (see Tables 8 and 9). Consequently, for most ionospheric applications it is possible to neglect the temperature dependence of the minor ion correction factors.

For practical computation, we note that $\Delta\left(x, \mathrm{H}^{+}\right)$ is negligibly small for all the ionospheric ions considered in Tables 8 and 9 , except $\mathrm{He}^{+}$over the range $n\left(\mathrm{O}^{+}\right) / n\left(\mathrm{H}^{+}\right)>4$. However, in this range minor ion collisions with $\mathrm{O}^{+}$dominate, and since $\Delta\left(x, \mathrm{H}^{+}\right)$ always appears as $v\left(x, \mathrm{H}^{+}\right)\left[1-\Delta\left(x, \mathrm{H}^{+}\right)\right]$its contribution can be neglected. Therefore, when evaluating the minor ion diffusion and frictional drag coefficients, an error of less than $10 \%$ is introduced by setting $\Delta\left(x, \mathrm{H}^{+}\right)=0$.

The frictional drag coefficients $h_{x i}$ and $h_{x j}$ account for the tendency of the minor ion to acquire the drift velocity of the major ions (Schunk and Walker, 1970a). For these coefficients, we note only that (1) by definition, $h_{x i}+h_{x j}=1$, (2) the quantity $\left(R_{i j}-R_{i i}\right)$ is negligibly small for all ionospheric ions and (3) these coefficients are not very sensitive to temperature.

Our final discussion deals with the heat flow equations (10) and (11). As noted previously, a temperature gradient in either major ion gas causes heat to flow in both gases. Also, a relative drift between the major ion gases causes heat to flow in both gases. The importance of these processes can be determined by comparing the terms in the heat flow equations. This comparison can be done, for example, by setting the relative flow term in the species heat flow equation separately equal to both major ion temperature gradient terms. With this procedure, we not only can determine which temperature gradient term is more important, but we also obtain the magnitude of the relative drift that produces the same heat flow as a given temperature gradient.

The results of such a comparison are shown in Table 10 for the case where $\mathrm{O}^{+}$and $\mathrm{H}^{+}$are the major ions. In generating this table, we set $\left.\mathrm{T}^{+} \mathrm{O}^{+}\right)=$ $T\left(\mathrm{H}^{+}\right)=1000 \mathrm{~K}, \quad \nabla T\left(\mathrm{O}^{+}\right)=\nabla T\left(\mathrm{H}^{+}\right)=1 \mathrm{~K} / \mathrm{km}$ and $n_{e}=5 \times 10^{4} \mathrm{~cm}^{-3}$. Since the drift velocity differences shown in Table 10 are proportional to $T\left(\mathrm{H}^{+}\right)^{3 / 2} \nabla T_{s} / n_{e}$, where $s=\mathrm{H}^{+}$or $\mathrm{O}^{+}$, they can be readily scaled to other values of these parameters. In the expression for $q\left(\mathrm{O}^{+}\right)$, larger $\mathrm{H}^{+}-\mathrm{O}^{+}$drift velocity differences are needed to match the $\nabla T\left(\mathrm{O}^{+}\right)$term than the $\nabla \mathrm{T}\left(\mathrm{H}^{+}\right)$term, indicating that
TABle 10. MAGNITUde OF THE $\mathrm{H}^{+}-\mathrm{O}^{+}$VELOCITY DIFFERENCE ( $\mathrm{m} / \mathrm{sec})$ THAT PRODUCES THE SAME HEAT FLOW AS THE INDICATED TEMPERATURE GRADIENT TERM. FOR THIS TABLE, $T\left(\mathrm{O}^{+}\right)=T\left(\mathrm{H}^{+}\right)=1000^{\circ} \mathrm{K}$, $\nabla T\left(\mathrm{O}^{+}\right)=\nabla T\left(\mathrm{H}^{+}\right)=1^{\circ} \mathrm{K} / \mathrm{km}$, AND $n_{e}=5 \times 10^{4} \mathrm{~cm}^{-3}$

\begin{tabular}{lrrrr}
\hline & \multicolumn{2}{|c|}{$g\left(0^{+}\right)$} & \multicolumn{2}{c}{$g\left(\mathrm{H}^{+}\right)$} \\
\hline $\mathrm{n}\left(0^{+}\right) / \mathrm{n}\left(\mathrm{H}^{+}\right)$ & $\nabla \mathrm{T}\left(0^{+}\right)$ & $\nabla T\left(\mathrm{H}^{+}\right)$ & $\nabla \mathrm{T}\left(0^{+}\right)$ & $\nabla T\left(\mathrm{H}^{+}\right)$ \\
\hline 0.001 & 119 & 30.6 & 0.41 & 7470 \\
0.01 & 116 & 29.2 & 0.41 & 754 \\
0.05 & 106 & 24.6 & 0.41 & 157 \\
0.1 & 99 & 20.8 & 0.41 & 82 \\
0.25 & 90 & 15.0 & 0.42 & 37 \\
0.5 & 90 & 11.2 & 0.43 & 22 \\
1.0 & 104 & 8.5 & 0.44 & 15 \\
2.0 & 141 & 6.8 & 0.45 & 11.2 \\
4.0 & 221 & 5.9 & 0.46 & 9.4 \\
10.0 & 466 & 5.3 & 0.47 & 8.2 \\
50.0 & 877 & 5.1 & 0.48 & 7.9 \\
100.0 & 4170 & 5.0 & 0.48 & 7.6 \\
1000.0 & 41,200 & 4.9 & 0.48 & 7.5 \\
& & & & \\
\hline
\end{tabular}

the $\nabla T\left(\mathrm{O}^{+}\right)$term is the dominant temperature gradient term when the $\mathrm{O}^{+}$and $\mathrm{H}^{+}$temperature gradients are comparable. However, the $\nabla T\left(\mathrm{H}^{+}\right)$term does make about a $20 \%$ contribution to $q\left(\mathrm{O}^{+}\right)$ when $\mathrm{O}^{+}$becomes a minor ion. This contribution is larger if $\nabla T\left(\mathrm{H}^{+}\right)>\nabla T\left(\mathrm{O}^{+}\right)$. In the expression for $\mathbf{q}\left(\mathrm{H}^{+}\right)$, on the other hand, the familiar $\mathrm{H}^{+}$temperature gradient term is much larger than the $\nabla T\left(\mathrm{O}^{+}\right)$ term for all $\mathrm{O}^{+} / \mathrm{H}^{+}$density ratios shown in Table 10. In order for an $\mathrm{O}^{+}$temperature gradient to affect $q\left(\mathrm{H}^{+}\right), \mathrm{H}^{+}$would have to be a minor ion and $\nabla T\left(\mathrm{O}^{+}\right)$would have to be 15 times greater than $\nabla T\left(\mathrm{H}^{+}\right)$. This situation is not likely to occur in the mid-latitude ionosphere.

With regard to the ion heat flow induced by an $\mathrm{H}^{+}-\mathrm{O}^{+}$relative drift, the column of larger numbers for a given $q$ indicates the relative drift that is needed for this process to match the heat flow induced by the dominant temperature gradient term. For $\mathrm{O}^{+}$, the field-aligned $\mathrm{H}^{+}-\mathrm{O}^{+}$relative drift would have to be greater than $100 \mathrm{~m} / \mathrm{sec}$ for this process to induce the same heat flow as a $1 \mathrm{~K} / \mathrm{km}$ $\mathrm{O}^{+}$temperature gradient. Larger relative flows are needed to match larger $\mathrm{O}^{+}$temperature gradients. For $\mathrm{H}^{+}$, on the other hand, an $\mathrm{H}^{+}-\mathrm{O}^{+}$relative drift of only $7.5 \mathrm{~m} / \mathrm{sec}$ induces an $\mathrm{H}^{+}$heat flow equivalent to a $1 \mathrm{~K} / \mathrm{km} \mathrm{H}^{+}$temperature gradient, if $\mathrm{H}^{+}$is a minor ion. For comparable $\mathrm{H}^{+}$and $\mathrm{O}^{+}$densities, a field-aligned relative drift of $15 \mathrm{~m} / \mathrm{sec}$ is equivalent to $1 \mathrm{~K} / \mathrm{km}$.

Recent measurements at Arecibo by Vickrey et al. (1976) indicate that field-aligned $\mathrm{H}^{+}-\mathrm{O}^{+}$relative drifts as large as $80 \mathrm{~m} / \mathrm{sec}$ occur in the transition region between the $F$ region and the 
protonosphere after sunset. Since the measured electron densities and ion temperatures are comparable to those adopted in our calculation, Table 10 indicates that the observed $80 \mathrm{~m} / \mathrm{sec}$ relative drift induces a significant $\mathrm{H}^{+}$heat flow and a detectable $\mathrm{O}^{+}$heat flow. Furthermore, the divergence or convergence of the induced heat flow probably has a significant effect on the $\mathrm{H}^{+}$energy balance in the transition region, but a negligible effect on the $\mathrm{O}^{+}$energy balance.

Additional evidence for the existence of large $\mathrm{H}^{+}-\mathrm{O}^{+}$relative drifts has been presented by Bailey et al. (1973) in their study of the nocturnal equatorial ionosphere. Model studies indicate that fieldaligned $\mathrm{H}^{+}-\mathrm{O}^{+}$relative drifts of up to $300 \mathrm{~m} / \mathrm{sec}$ are possible due to the asymmetry in the summer and winter hemispheres which results from meridional neutral winds and electrodynamic drifts. When the values in Table 10 are scaled using the $\mathrm{O}^{+}$and $\mathrm{H}^{+}$densities calculated by Bailey et al. (1973), we find that $\mathrm{H}^{+}-\mathrm{O}^{+}$relative drifts of this order of magnitude are large enough to affect significantly the ion heat fiow in the nocturnal equatorial ionosphere. This additional induced heat flow, in turn, may alter the ion energy balance in this region.

As noted earlier, $\mathrm{H}^{+}-\mathrm{O}^{+}$relative drifts have their greatest effect on the ion heat flow when the relative drift is large and $\mathrm{H}^{+}$is a minor ion. Such conditions exist at high latitudes in the polar wind where $\mathrm{H}^{+}$flows through $\mathrm{O}^{+}$at speeds approaching several $\mathrm{km} / \mathrm{sec}$. Unfortunately, our diffusion and heat flow equations are not valid in this region, since we have assumed that the species temperature and flow velocity differences are small. Nevertheless, the relative flow that exists between $\mathrm{H}^{+}$and $\mathrm{O}^{+}$in the polar wind could induce a significant $\mathrm{H}^{+}$ heat flow and this, in turn, could alter the $\mathrm{H}^{+}$ energy balance.

\section{SUMMARY}

We have derived diffusion and heat flow equations for the mid-latitude topside ionosphere in an approximation that is equivalent to the second order multicomponent formulation of Hirschfelder et al. (1964). However, our equations have an advantage over those obtained from the multicomponent formulation in that each constituent of the gas mixture is allowed to have its own temperature and drift velocity.

Considering two major ions, electrons, and a number of minor ions, we have found that a heat flow through both major ion gases results from a temperature gradient in either gas or from a relative drift between the major ion gases [see equations (10) and (11)]. The corresponding major ion thermal conductivities, $\lambda_{3}$, and thermoelectric coefficients, $\delta_{s t}$, are given by equations (13) and (14). We have also found that a temperature gradient in either of the major ion gases or in the electron gas causes thermal diffusion in both major ion gases [see equations (18) and (19)]. The corresponding thermal diffusion coefficients $\alpha_{i j}, \alpha_{i j}{ }^{*}, \gamma_{i}$ and $\gamma_{i}$ are given by equations (22)-(25). The major ion ordinary diffusion coefficients $D_{1}$ and $D_{1}$ are given by equations (20) and (21). The quantity $\Delta_{i j}$, which appears in the expressions for the ordinary diffusion coefficients, accounts for the effect of heat flow on ordinary diffusion and is given by equation (26).

As far as the minor ion is concerned, we have found that a heat flow through the minor ion gas can result from a temperature gradient in either of the major ion gases, a temperature gradient in the minor ion gas, a relative drift between the minor ion gas and either of the major ion gases, or a relative drift between the major ion gases [see equation (30)]. The appropriate minor ion transport coefficients are given by equations (31)-(33).

The motion of the minor ion is influenced by the motion of both major ions in addition to ordinary and thermal diffusion processes [see equation (34)]. The tendency of the minor ion to be dragged along by the major ions enters through the frictional drag coefficients $h_{x i}$ and $h_{x i}$ [equation (38)]. The ordinary diffusion of the minor ion is described by the coefficient $D_{x}$ [equation (35)]. Thermal diffusion in the minor ion gas can result from a temperature gradient in the minor ion gas, in either of the major ion gases, or in the electron gas. The corresponding thermal diffusion coefficients $\beta_{x}, \beta_{i}, \beta_{j}$ and $\gamma_{x}$ are given by equations (36) and (37).

We have evaluated the importance of some of the new transport processes. For the case where $\mathrm{O}^{+}$ and $\mathrm{H}^{+}$are the major ions, we have found that:

(1) Thermal diffusion of both $\mathrm{O}^{+}$and $\mathrm{H}^{+}$is governed by the $\mathrm{H}^{+}$temperature gradient.

(2) The increase in the $\mathrm{H}^{+}$and $\mathrm{O}^{+}$diffusion coefficients that results from the coupling between diffusion and heat flow is greatest when $\mathrm{H}^{+}$is a minor ion. In this limit the $\mathrm{H}^{+}$and $\mathrm{O}^{+}$diffusion coefficients are increased by a factor of 2.76 .

(3) $\mathrm{An}^{+}$temperature gradient induces a negligibly small amount of $\mathrm{H}^{+}$heat flow.

(4) An $\mathrm{H}^{+}$temperature gradient induces a significant amount of $\mathrm{O}^{+}$heat flow when $\nabla T\left(\mathrm{H}^{+}\right) \geq$ $\nabla T\left(\mathrm{O}^{+}\right)$and $\mathrm{H}^{+}$is the more abundant major ion. 
(5) A relative drift between $\mathrm{H}^{+}$and $\mathrm{O}^{+}$induces a heat flow in both gases, but it is more important for $\mathrm{H}^{+}$than $\mathrm{O}^{+}$. This induced $\mathrm{H}^{+}$heat flow could significantly affect the $\mathrm{H}^{+}$energy balance in the nocturnal equatorial ionosphere and in the midlatitude ionosphere after sunset in a region between the $F$ region and the protonsphere.

Finally, we note that several simplifying assumptions are implicit in our diffusion and heat flow equations. In deriving these equations, we have assumed a steady state, neglected density and temperature gradients perpendicular to magnetic field lines, neglected stress and nonlinear acceleration terms, and used Burgers' linear collision terms. The use of Burgers' linear collision terms implies small temperature and flow velocity differences between the interacting species.

Acknowledgements-This research was supported, in part, by NSF Grant ATM74-21090 A02 and Air Force Grant F19628-77-G-0007 at the University of Michigan, by NSF Grant DES74-22343 at the University of California, San Diego, and by NSF Grant ATM76-19792 at Utah State University.

\section{REFERENCES}

Bailey, G. J., Moffett, R. J., Hanson, W. B. Sanatani, S. (1973). Effects of interhemisphere transport on plasma temperatures at low latitudes. J. geophys. Res. 78, 5597.

Banks, P. M., Nagy, A. F. and Axford, W. I. (1971). Dynamical behavior of thermal protons in the midlatitude ionosphere and magnetosphere. Planet. Space Sci. 19, 1053.

Bauer, S. J. (1966). The structure of the topside ionosphere. In Electron Density Profiles in the Ionosphere and Exosphere (Ed. J. Frihagn). North-Holland, Amsterdam.

Behnke, R. A. (1970). Vector measurements of the ion transport velocity with applications to $F$-region dynamics. Ph.D. thesis, Rice University, Houston, Texas.

Burgers, J. M. (1969). Flow Equations for Composite Gases. Academic Press, New York.

Chapman, S. and Cowling, T. G. (1970). The Mathematical Theory of Non-Uniform Gases. Cambridge University Press, London.

Devoto, R. S. (1966). Transport properties of ionized monoatomic gases. Phys. Fluids 9, 1230.

Evans, J. V. (1969). Theory and practice of ionosphere study by Thomson scatter radar. Proc. IEEE 57, 496.

Evans, J. V. (1971a). Observations of $F$-region vertical velocities at Millstone Hill: I. Evidence for drifts due to expansion, contraction and winds. Radio Sci. 6, 609.

Evans, J. V. (1971b). Observations of F-region vertical velocites at Millstone Hill: II. Evidence for fluxes into and out of the protonosphere. Radio Sci. 6, 843.

Evans, J. V., Julian, R. F. and Reid, W. A. (1970). Incoherent scatter measurements of $F$-region density, temperatures and vertical velocity at Millstone Hill. M.I.T. Lincoln Lab. Rep. 477.
Grad, H. (1949). On the kinetic theory of rarefied gases. Commun. Pure appl. Math. 2, 331.

Grad, H. (1958). Principles of the kinetic theory of gases. Handb. Phys. 12, 205.

Hagen, J. B. (1972). A hybria autocorrelator and its application to high altitude incoherent scatter. Nat. Astro. Ionosphere Center Arecibo, Puerto Rico, Rep. 18.

Hirschfelder, J. O., Curtiss, C. F. and Bird, R. B. (1964). Molecular Theory of Gases and Liquids. John Wiley, New York.

Johnson, F. S. (1960). The ion distribution above the $F_{2}$-maximum. J. geophys. Res. 65, 577.

Kockarts, G. and Nicolet, M. (1963). Ann. Geophys. 19, 370.

Mange, P. (1960). The distribution of minor ions in electrostatic equilibrium in the high atmosphere. $J$. geophys. Res. 65, 3833.

Massa, J. L., Cicerone, R. J. and Nagy, A. F. (1974). Theoretical and experimental studies of ionization exchange between the ionosphere and plasmasphere. Paper presented at the Int. Symp. Solar Terrestrial Physics, Saõ Paulo, Brazil. Summary in Atmosphere 3, 338.

Mayr, H. G., Fontheim, E. G., Brace, L. H., Brinton, H. C. and Taylor, H. A. (1972). A theoretical model of the ionosphere dynamics with interhemispheric coupling. J. atmos. terr. Phys. 34, 1659.

Meador, W. E. and Staton, L. D. (1965). Electrical and thermal properties of plasmas. Phys. Fluids 8, 1694.

Moffett, R. J. and Murphy, J. A. (1973). Coupling between the $F$-region and protonosphere: numerical solution of the time-dependent equations. Planet. Space Sci. 21, 43.

Murphy, J. A., Bailey, G. J. and Moffett, R. J. (1977). Calculated diurnal variations of $\mathrm{O}^{+}$and $\mathrm{H}^{+}$at midlatitudes: I. Protonospheric replenishment and F-region behavior at sunspot minimum. J. atmos. terr. Phys. To be published.

Nagy, A. F. and Banks, P. M. (1972). Diurnal variation of the $\mathrm{H}^{+}$flux between the ionosphere and the protonosphere. J. geophys. Res. 77, 4277.

Roble, R. G. and Hastings, J. T. (1977). Thermal response properties of the Earth's ionospheric plasma. Planet. Space Sci. 25, 217.

Schunk, R. W. (1975). Transport equations for aeronomy. Planet Space Sci. 23, 437.

Schunk, R. W. and Walker, J. C. G. (1969). Thermal diffusion in the topside ionosphere for mixtures which include multiply-charged ions. Planet. Space Sci. 17, 853.

Schunk, R. W. and Walker, J. C. G. (1970a). Thermal diffusion in the $F_{2}$-region of the ionosphere. Planet Space Sci. 18, 535 .

Schunk, R. W. and Walker, J. C. G. (1970b). Minor ion diffusion in the $F_{2}$-region of the ionosphere. Planet. Space. Sci. 18, 1319.

Schunk, R. W. and Walker, J. C. G. (1970c). Transport properties of the ionospheric electron gas. Planet. Space Sci. 18, 1535.

Shkarofsky, I. P. (1961). Values of the transport coefficients in a plasma for any degree of ionization based on a Maxwellian distribution. Can. J. Phys. 39, 1619.

Spitzer, L. and Härm, R. (1953). Transport phenomena in a completely ionized gas. Phys. Rev. 89, 977.

Vasseur, G., and Waldteufel, P. Etude par diffusion de 
Thomson de la production et de la recombinaison dans la region-F de l'ionosphere diurne. J. atmos. terr. Phys. 30, 779.

Vickrey, J. F., Swartz, W. E. and Farley, D. T. (1976). Incoherent scatter measurements of ion counterstreaming. Geophys. Res. Lett. 3, 217.

Walker, J. C. G. (1967). Thermal diffusion in the topside ionosphere. Planet. Space Sci. 15, 1151.

\section{APPENDIX}

The effective collision frequencies are given by

$$
\begin{aligned}
& \nu_{i}^{\prime}=\nu_{i i}+\frac{5}{4} \nu_{i j}\left[D_{i j}^{(1)}+\frac{3}{2} \frac{\mu_{i j}}{m_{i}} \frac{T_{i}}{T_{i j}}\right] \\
& v_{j}^{\prime}=v_{j j}+\frac{5}{4} v_{j i}\left[D_{j i}^{(1)}+\frac{3}{2} \frac{\mu_{i i}}{m_{j}} \frac{T_{j}}{T_{j i}}\right] \\
& y_{i j}^{\prime}=\frac{5}{4} v_{i j}\left[D_{i j}^{(4)}+\frac{3}{2} \frac{\mu_{i j}}{m_{i}} \frac{T_{l}}{T_{i j}}\right] \\
& \nu_{j i}{ }^{\prime}=\frac{5}{4} \nu_{j i}\left[D_{j i}^{(4)}+\frac{3}{2} \frac{\mu_{i l}}{m_{j}} \frac{T_{l}}{T_{j i}}\right] \\
& v_{e}^{\prime}=v_{e e}+\frac{13}{8}\left(v_{e i}+v_{e j}\right) \\
& \nu_{x i}{ }^{\prime}=\frac{3}{4} \nu_{x i}\left[D_{x i}^{(4)}+\frac{3}{2} \frac{\mu_{x i}}{m_{x}} \frac{T_{x}}{T_{x i}}\right] \\
& \nu_{x i}{ }^{\prime}=\frac{5}{4} \nu_{x j}\left[D_{x j}^{(4)}+\frac{3}{2} \frac{\mu_{x j}}{m_{x}} \frac{T_{x}}{T_{x j}}\right] \\
& \nu_{x}^{\prime}=\frac{5}{4} \sum_{s=i, j} \nu_{x s}\left[D_{x s}^{(1)}+\frac{3}{2} \frac{\mu_{x x}}{m_{x}} \frac{T_{x}}{T_{x s}}\right] \text {. }
\end{aligned}
$$

The dimensionless quantities appearing in the minor ion diffusion equation are given by

$$
\begin{aligned}
& Q_{i i}=1-\frac{\nu_{x i}^{\prime}}{\nu_{x}^{\prime}}-\frac{p_{i} \nu_{x i}^{\prime}}{a_{i} \nu_{x}^{\prime}} \\
& Q_{i j}=Q_{i l}-1+\frac{\nu_{j i}^{\prime}}{\nu_{j}^{\prime}} \\
& Y_{i i}=1-\frac{\nu_{x i}^{\prime}}{\nu_{x}^{\prime}}-\frac{\nu_{i j}^{\prime}}{\nu_{i}^{\prime}} \frac{\nu_{x j}^{\prime}}{\nu_{x}^{\prime}} \\
& Y_{i j}=Y_{i}-1+\frac{\nu_{j i}^{\prime}}{\nu_{i}^{\prime}} \\
& Y_{x i}=Y_{x j}=-(1-\xi) .
\end{aligned}
$$

The quantities $Q_{i j}, Q_{i j}, Y_{i j}$ and $Y_{j i}$ can be obtained from the above equations by interchanging the subscripts $i$ and $j$.

It should be noted that the Coulomb collision frequencies are not symmetric but satisfy the relation $n_{3} m_{3} \nu_{31}=$ $n_{t} m_{t} v_{t s}$. For in $\Lambda=15$, the Coulomb coliision frequency (3) can be approximated numerically by

$$
v_{s t}=1.27 \frac{z_{s}^{2} z_{t}^{2} A_{s t}^{1 / 2}}{A_{s}} \frac{n_{t}}{T_{s t}^{3 / 2}},
$$

where $A_{3}$ is the particle mass in atomic mass units, $A_{s}$ is the reduced mass in atomic mass units, $n_{1}$ is in $\mathrm{cm}^{-3}$ and $T_{s t}$ is in $K$. For electron-electron and electron-ion collisions, the Coulomb collision frequency reduces further to

$$
\begin{aligned}
& \nu_{e i}=54.5 \frac{m_{i} z_{i}^{2}}{T_{e}^{3 / 2}} \\
& \nu_{e e}=\frac{54.5}{\sqrt{2}} \frac{n_{e}}{T_{e}^{3 / 2}} .
\end{aligned}
$$

